\title{
Switchback-like structures observed by Solar Orbiter
}

\author{
A. Fedorov ${ }^{1}$, P. Louarn ${ }^{1}$, C. J. Owen ${ }^{2}$, T. S. Horbury ${ }^{3}$, L. Prech ${ }^{4}$, T. Durovcova ${ }^{4}$, A. Barthe ${ }^{1}$, A. P. Rouillard ${ }^{1}$, \\ J. C. Kasper ${ }^{5,6}$, S. D. Bale ${ }^{7,8}$, R. Bruno ${ }^{9}$, H. O’Brien ${ }^{3}$, V. Evans ${ }^{3}$, V. Angelini ${ }^{3}$, D. Larson ${ }^{8}$, R. Livi ${ }^{8}$, B. Lavraud ${ }^{1,10}$, \\ N. Andre ${ }^{1}$, V. Genot ${ }^{1}$, E. Penou ${ }^{1}$, G. Mele ${ }^{11}$, and V. Fortunato ${ }^{12}$
}

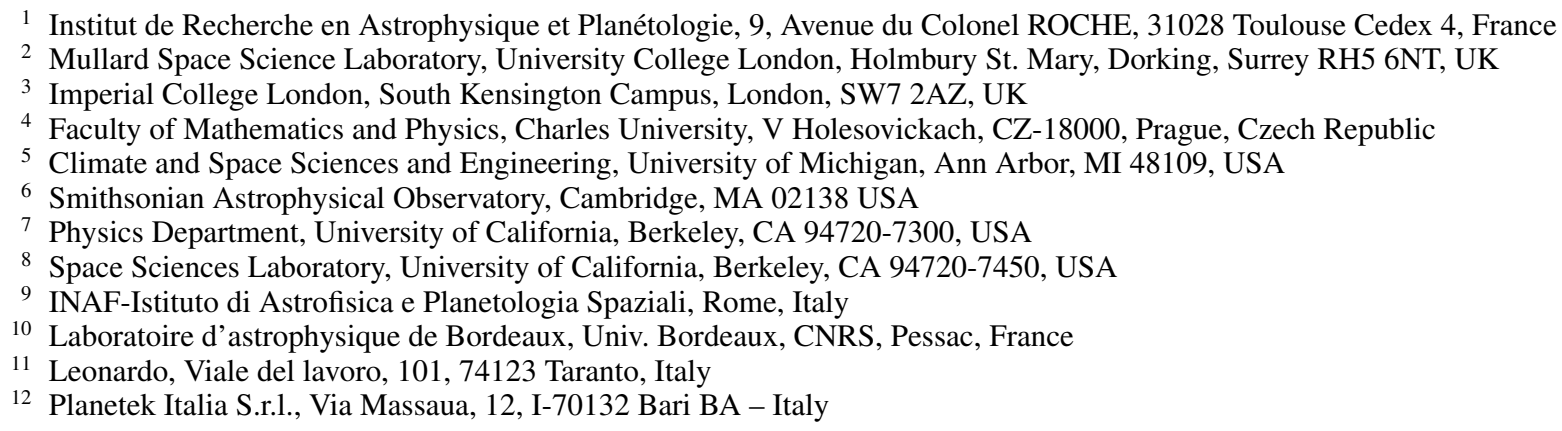

Received XX XXX XX Accepted XX XXX XX

\begin{abstract}
Context. Rapid polarity reversals of the radial heliospheric magnetic field were discovered by Ulysses and they are now frequently observed as a common near-Sun phenomenon by NASA's Parker Solar Probe (PSP). Other solar wind missions, including ESANASA Solar Orbiter (SolO), also observe similar phenomena. The nature of these fluctuations is unclear, and the relation between the "switchbacks" observed near the Sun and similar events observed at $1 \mathrm{AU}$ is unknown.

Aims. We make a detailed case study of the SolO plasma and magnetic field data obtained in a region that is magnetically connected to a coronal hole. We aim to check whether such fluctuations might have the same origin as the "switchbacks" observed by PSP. Methods. We used PSP magnetic field and plasma data to support our analysis of SolO data. We used the magnetic field data, electron pitch angle distributions, proton velocity distribution functions, and $\alpha$-particle parameters measured by SolO to perform a detailed analysis of the observed solar wind perturbations.

Results. On 27 September 2020, PSP and SolO were located around the same Carrington longitude and their latitudinal separation was very small. Solar wind plasma and magnetic field data during this time interval confirms that (at least at certain times) the solar wind observed by both spacecraft does originate from the same coronal hole region and that during these time intervals, SolO experiences several short variations similar to the "switchbacks" regularly observed by PSP.

Conclusions. The suprathermal electron pitch angle distributions and $\alpha$-particle speed variations indicate that the magnetic field line was bent by $180^{\circ}$ by solar wind velocity shear. Variations in electron and proton velocity distribution functions suggest that bent field lines reconnect with each other, producing flux ropes. The observed flux ropes might be the surviving and modified remains of the switchbacks created near Sun and observed by PSP.
\end{abstract}

Key words. Heliosphere - Solar wind - Reconnection - Switchback

\section{Introduction}

In 1995-1996, during the solar minimum, the Ulysses spacecraft, orbiting at high heliolatitudes, observed numerous radial magnetic field polarity inversions (see Balogh et al. 1999) in the fast solar wind that is magnetically mapped to the polar coronal hole. The field reversals tend to occur very rapidly and the magnetic field direction, opposite to the dominant polarity, is observed almost eight percent of the time. Subsequently, similar structures were observed by near-Earth heliospheric spacecrafts such as ACE (see Owens et al. 2013). Decades later, the first orbit of NASA's Parker Solar Probe (PSP) (Fox et al. 2016), in November 2018, led to the discovery of a similar, but unexpected feature of the near-Sun solar wind: the presence of frequent and rapid polarity reversals of the radial magnetic field (Bale et al. 2019). These reversals, called "switchbacks," were accompanied by strong fluctuations of the solar wind velocity vector (Kasper et al. 2019). The nature and origin of these "switchbacks" are not clear at the moment. Thus, while PSP observations have shown an intense "switchback" activity at distances around and below $0.3 \mathrm{AU}$, similar events also have been observed at distances of 1 AU (Kahler et al. 1996; Gosling et al. 2011) and even all the way to 2.9 AU (Yamauchi et al. 2002). The question of whether all such observations relate to the same phenomenon is still open.

The launch of the partner mission Solar Orbiter (SolO), a space mission made possible through an international collaboration between ESA and NASA, operated by ESA; for more see (Muller, D. et al. 2020; García Marirrodriga, C. et al. 2021) potentially opens a promising opportunity for coordinated studies of the heliosphere phenomena, including switchbacks. This possibility will become feasible during the SolO science phase, starting at the end of 2021. During the SolO cruise phase, we have very little opportunity for such collaborations, however, 

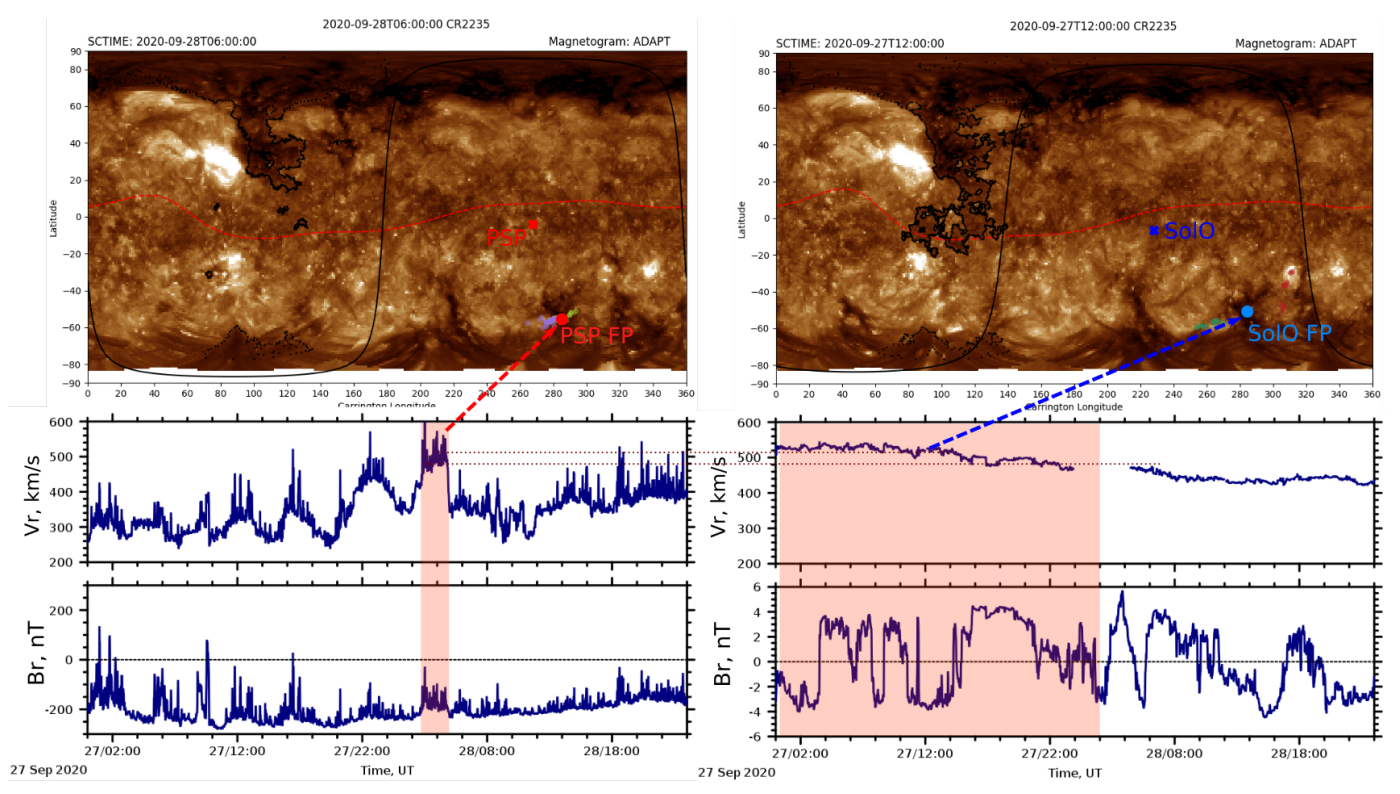

Fig. 1. Summary of the PSP and SolO observations between 27th and 28th September 2020 when both spacecrafts have been magnetically connected to the same coronal hole. Top: Synoptic map produced by the connectivity tool (Rouillard, A. P. et al. 2020) overlayed on a ADAPT/GONG magnetogram, and on a STEREO-A/EUVI 193 A Carrington map. The map is obtained for Carrington rotation \#2235. Left: Red cross shows the PSP sub-spacecraft point, and the red circle shows the PSP expected footprint along the magnetic field line. The connectivity is calculated for the time interval indicated by the pink band in the bottom panel. Right: Same details as in the left panel but for SolO. Bottom: Radial proton velocity and the radial component of the magnetic field measured by PSP (left) and SolO (right).

from time to time, SolO observes the fast inversions of the magnetic field. It would be useful for us to know what PSP would see during the encounter phase, at the field line originating from the same coronal region within a reasonable time window. Fortunately, we have found such a case. Thus, the purpose of the present paper is to carefully analyze the solar wind perturbations observed by SolO when the spacecraft is in a plasma flow that is magnetically connected to the region also observed by PSP somewhat earlier. For the present study, we make use of some very general information obtained from PSP.

Here, we use the data from the relevant instruments as follows: the PSP FIELDS package (Bale et al. 2016), which provides the magnetic field measurements up to 290 samples per second; the PSP SWEAP (Solar Wind Electrons Alphas and Protons) instrument suite (Kasper et al. 2016), including the Solar Probe Cup (SPC) and the Solar Probe Analyzers (SPAN), which provides solar wind parameters with up to $4 \mathrm{~Hz}$ cadence; the SolO MAG (Horbury, T. S. et al. 2020) fluxgate magnetometer providing $8 \mathrm{~Hz}$ magnetic field measurements; and SolO SWA (Solar Wind Analyzer) (see Owen, C. J. et al. 2020), providing the electron (EAS sensor), proton, and $\alpha$-particle (PAS sensor) $3 \mathrm{D}$ velocity distribution functions (VDF), with up to $4 \mathrm{~Hz}$ in resolution.

Horbury et al. (2021) found that PSP and SolO were connected to the same coronal hole between 27-28 September 2020. They are linked to this coronal region at different times and thus we cannot say that both spacecraft have observed the same plasma, but they have observed solar wind ejected from the same coronal hole at fairly close time intervals. As shown in Figure 1, in the lightly red shaded intervals, PSP observed a rather high speed solar wind similar to that observed by SolO when connected to the same coronal hole. Even the speed profiles measured by both spacecraft are similar. We note that SolO crosses the coronal hole from high to low Carrington longitudes. This is natural since the Carrington rotation is faster than the SolO orbital motion. By contrast, PSP moves quickly from low to high
Carrington longitudes; thus, in the Carrington coordinate frame the two spacecraft fly toward each other.

Figure 1 shows the time intervals when both spacecraft observed similar solar wind speeds and were magnetically connected to the same coronal hole. Since PSP measures solar wind that would not reach SolO for several days, the similar velocity profiles on both spacecrafts tell us that we see a quasi steadystate spacial structure of the coronal hole. However, in comparing other solar wind parameters observed by the two spacecraft, we see significant differences. PSP has observed a strong negative radial magnetic field with significant fluctuations. But these fluctuations never change the polarity of the $B_{r}$ component. The solar wind $V_{r}$ is also strongly fluctuating. By contrast, during that period, SolO has observed complete reversals of the radial component of magnetic field, but along a rather stable $V_{r}$.

In this paper, we present a detailed analysis of SolO data and propose a simple scenario for these observations. Since we are extensively using the first data from the Solar Orbiter SWAPAS (Solar Wind Analyzer - Proton Alpha Sensor) instrument, our additional goal is to present the advantages of such modern measurements.

\section{Observations and preliminary data analysis}

\subsection{Observations overview}

The PSP measurements show that the heliospheric magnetic field was sunward at the source. The SolO data (presented in Figure 1) show that the sunward directed magnetic field occupies only $50 \%$ of the time interval mapped to the south hemisphere coronal hole. Since the plasma and magnetic field properties at 3:00, 8:00, and 12:30 UT (when the radial magnetic field is sunward) are very similar, we limit our analysis to the interval from 9:00 to 16.30 UT. Figure 2 shows a general view of magnetic field and ion measurements by SolO. The solar wind speed is slowly decreasing during this time interval, while the proton 


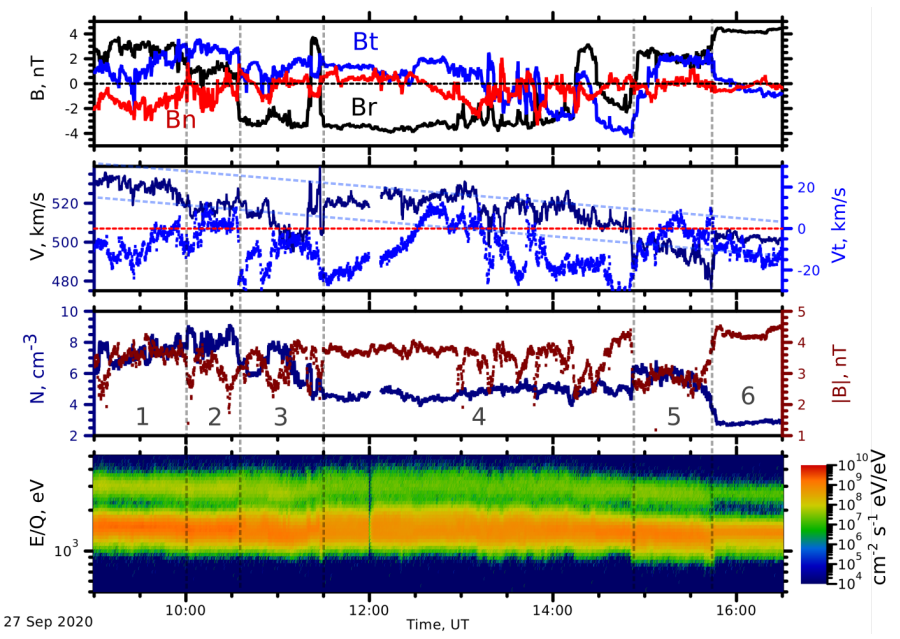

Fig. 2. SolO measurements. From top to bottom: Magnetic field in RTN frame, full proton velocity (navy) and tangential velocity (blue), number density (navy), and the magnetic field magnitude (blue), E/Q-time spectrogram of the differential ion energy flux. The vertical dashed lines marked the regions boundaries, explained in the text. The dashed light blue lines, in the second from the top panel, show the trend of maximal and minimal solar wind velocities. The plot is divided into six time intervals, as discussed in the text.

density is also decreasing but in steps. We note that we observe the "normal" sunward magnetic field in the time interval 10:30 15:00, labeled in Figure 2 as \#3 and \#4. This almost continuous sunward segment is interrupted twice by short "switchbacks" at $11: 30$ and at 14:15.

Another important data set is presented in Figure 3. It shows in the middle panel the pitch angle distribution (PAD) of suprathermal electrons. This population includes the "strahl" electrons that carry the heat flux away from Sun, and is thus always directed anti-sunward along open heliospheric field line (Feldman et al. 1975). These electrons directly inform us on the polarity of the magnetic field lines at the source, even if, locally, the field lines may be bent or even reversed (Owens et al. 2017). An important information about magnetic field topology can be derived also from the relative velocity of $\alpha$-particles ( $H e^{++}$ions) $V_{\alpha p}$ (see Yamauchi et al. 2004b). This tool allows us to corroborate the folded configuration of the field lines. The $\alpha$-particle relative velocity is calculated as : $V_{\alpha p}=\left|\boldsymbol{V}_{\boldsymbol{H e + +}}-\boldsymbol{V}_{\boldsymbol{H +}}\right| \cdot \operatorname{sign}\left(V_{\mathrm{He++}}^{R}-\right.$ $\left.V_{H+}^{R}\right)$. Generally speaking, $V_{\alpha p}$ is positive in the undisturbed fast solar wind (see Marsch et al. 1982; Reisenfeld et al. 2001), while negative $V_{\alpha p}$ indicates a reversed magnetic field line. We discuss this method in detail in Section 3.

In looking at Figures 2 and 3, we see a generally symmetric scene. Before 10:00 UT (region "\#1") and after 15:30 UT (region "\#6"), the magnetic field is radial and antisunward. We note that suprathermal "strahl" electrons are antiparallel there (i.e., moving toward the Sun); thus, the corresponding field lines are sunward at the source. This feature confirms the statement that SolO and PSP are magnetically connected to the same coronal hole. The $\alpha$-particles velocity is a bit lower than the proton velocity. Regions "\#2" and "\#5" appear as transition layers between the anti-sunward and sunward magnetic field directions and a strahl dropout is observed during these time intervals. The tangential magnetic field, combined with decreased magnetic field magnitude and increased density are a signature of a reconnection exhaust (see Enžl et al. 2014), albeit the solar wind velocity is dropping down at both intervals. The intervals of sunward magnetic field (regions \#3 and \#4) end with short "switchbacks."

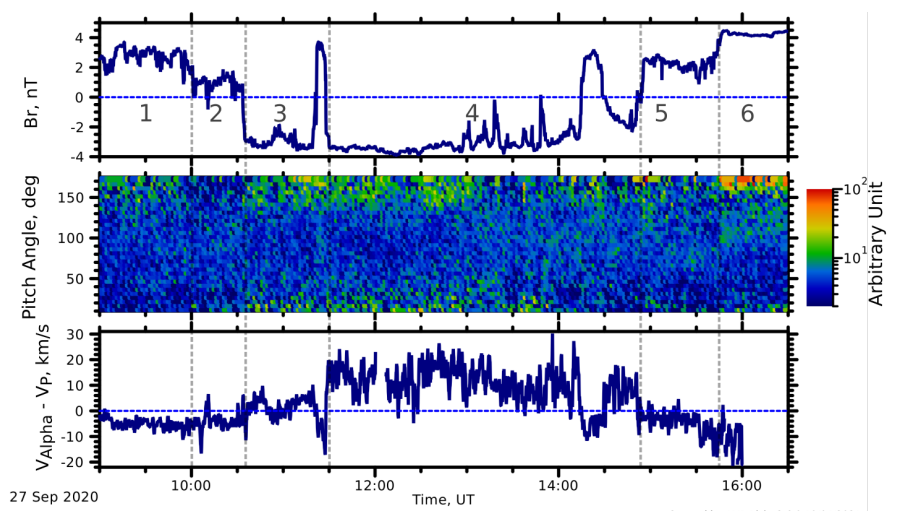

Fig. 3. Supporting information to Figure 2. From top to bottom: Radial magnetic field and pitch angle distribution (PAD) of the electrons: $E>$ $75 \mathrm{eV}, V_{\alpha p}$

$V_{\alpha p} \approx 0$ in region "\#3", and becomes positive and reaches about half of the local Alfven speed ( $30-40 \mathrm{~km} / \mathrm{s}$ ) in the time interval "\#4." An exciting feature observed in regions "\#3" and “\#4" is the signature (albeit weak) of bi-directional strahl electrons with a slight dominance of antiparallel fluxes. This phenomena is discussed in Section 3.

We note that the time interval "\#4" exhibits most of the properties of a flux rope: slowly rotating $B_{t}$ from positive to negative (Figure 2, top panel) and low $\beta$ (Lepping et al. 1990).

The embedded switchback observed at 11:25 UT is shown in Figure 4. The radial magnetic field is reversing for about 6 minutes without any significant perturbations in the other components. Interestingly, we see boundary layers on both sides of this switchback. Both boundary layers show abrupt increases of the proton bulk velocity as well as anti-correlated profiles of the number density and magnetic field magnitude. The changes in velocity, with jets, combined with decreased magnetic field and increased density, are all consistent with these boundary layers being the result of reconnection (see Gosling et al. 2005). The properties of the proton velocity distribution functions (VDF) are also consistent with reconnection. Before the first boundary layer (Figure 4, VDF a) we see proton distributions with $T_{\|}<T_{\perp}$. As soon as SolO enters the boundary layer, we see the VDF (b), with $T_{\|}>T_{\perp}$ and likely containing two populations. Such a VDF is typical for the reconnection exhaust due to the mixing of two solar wind populations from the two sides of the exhaust (see Gosling et al. (2005) and the discussion in Section 3). We note that reconnection has been observed at the boundaries of switchbacks with PSP also (Froment et al. 2021). In the center of the switchback, where the magnetic field is positive and radial (VDF c), the VDF again shows $T_{\|}<T_{\perp}$. Upon the exit of the switchback, the same features are repeated but in opposite order (distributions d and e). The multi-component nature of the proton VDF is particularly evident in distribution (e). When SolO enters region \#4, with negative $B_{r}$, the VDF becomes more smooth, but maintaining $T_{\|}>T_{\perp}$.

At the end of region \#4 (see Figure 2), we see several $B_{r}$ fluctuations that also show bipolar variations in $B_{t}, B_{n}$, as well as a reduced magnetic field pressure in equilibrium with the protons pressure. Figure 5 shows a zoom on one such case. Thus, we see a passage of a compressible structure and it is consistent with the occurrence of reconnection somewhere in the vicinity. We note a strahl dropout during such $B_{r}$ fluctuations. These magnetic field perturbations do no lead to the modification of the proton VDF. The ion distribution simply follows the instant direction of $\boldsymbol{B}$ vector. 


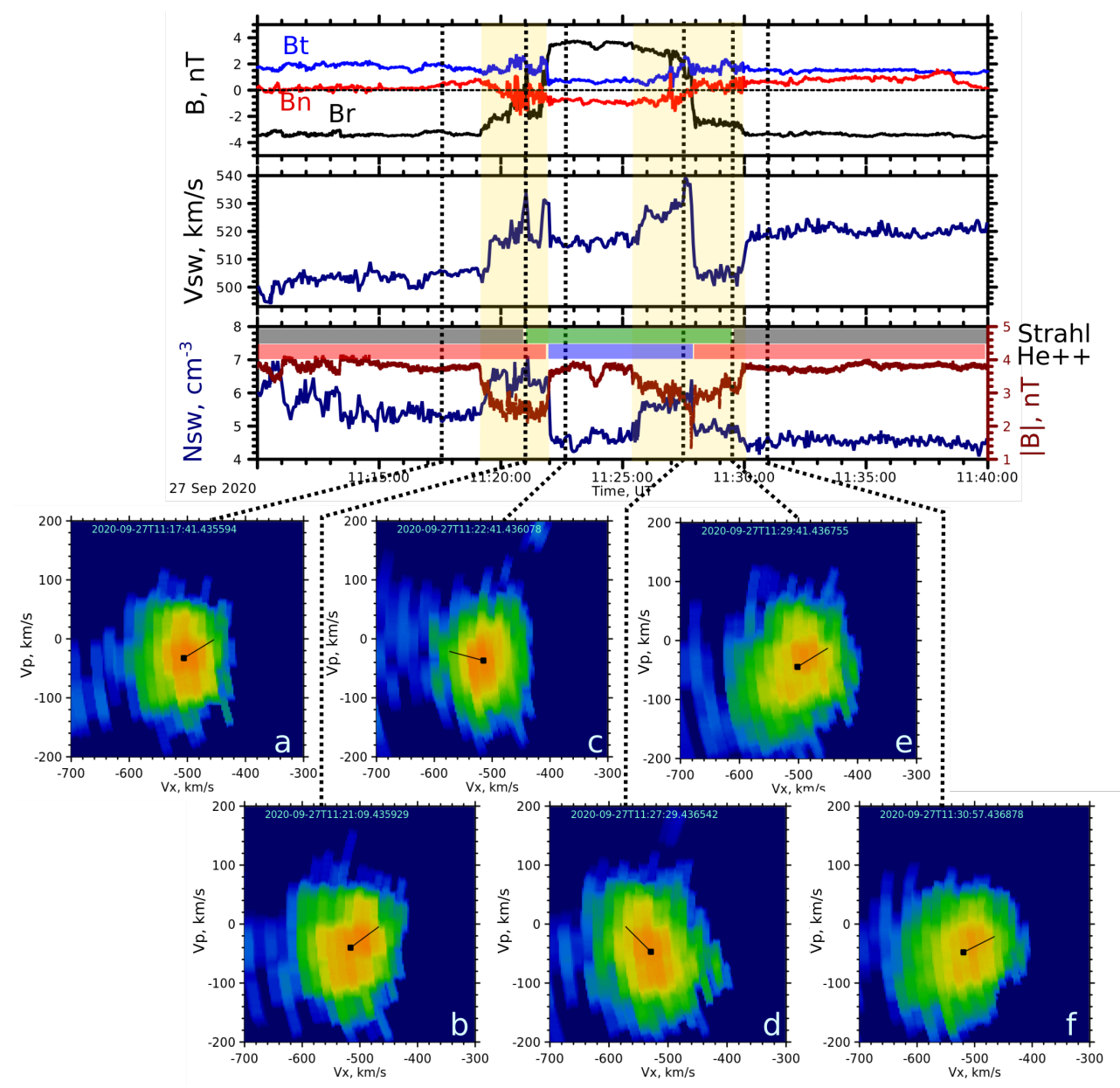

Fig. 4. Plasma properties around the short excursion of the magnetic field from the sunward $\left(B_{R}<0\right)$ to the antisunward. From top to bottom: Magnetic field, protons bulk velocity, protons density with the magnetic field magnitude, and proton velocity distribution functions (VDF) in the plane containing vectors $\boldsymbol{V}$ and $\boldsymbol{B}$. The horizontal axis $V_{X}$ in the VDF panels is an axis in the $\{\boldsymbol{V}, \boldsymbol{B}\}$ plane maximally parallel to the spacecraft $X$ axis. The vertical axis $V_{P}$ is perpendicular to $V_{X}$ in the plane. The horizontal bars in the panel of proton's number density show the time intervals of antiparallel strahl electrons in green and the intervals of the positive $V_{\alpha p}\left(V_{H e++}>V_{H_{+}}\right)$in red. The gray part of the strahl bar shows the bi-directional PAD. The blue bar shows the negative $V_{\alpha p}\left(V_{H e++} \leq V_{H+}\right)$.

\section{Discussion, a plausible scenario, and conclusions}

\subsection{Summary of the event}

In Section 2, we showed that in the time interval between 9:00 - 16:00 UT on 27 September 2020, SolO was magnetically connected to a coronal hole located in the southern hemisphere. The spacecraft was very far from the heliospheric current sheet and streamer belt. During this interval, we noted large-scale changes from the antisunward radial magnetic field to the sunward magnetic field and back. SolO mostly observed antiparallel strahl electrons and the solar wind velocity was slowly decreasing during the whole interval (Figure 2). We divided this time interval into six regions, as summarized below:

1. Anti-parallel strahl electrons, negative $V_{\alpha p}$, almost radial antisunward magnetic field, medium solar wind speed.

2. Strahl dropout, tangential magnetic field, combined with decreased magnetic field and increased density, proton distributions with $T_{\|} \simeq T_{\perp}, V_{\alpha p}=0$, lowest solar wind speed.

3. Sunward radial magnetic field, bi-directional strahl, proton distributions with $T_{\|}<T_{\perp}$ (with a small high energy tail),
$V_{\alpha p}>0$, and lowest solar wind speed at that time. A magnetic field reversal framed by two reconnecting boundary layers is observed at the end of that interval.

4. Sunward radial magnetic field turning to the tangential direction, bi-directional strahl, proton distributions with $T_{\|}>T_{\perp}$ containing two components, and maximum solar wind speed. Moderate pressure-balanced fluctuations with reconnection signatures are observed at the end of the interval.

5. Strahl dropout, tangential magnetic field, proton distributions with $T_{\|} \simeq T_{\perp}, V_{\alpha p}=0$ (similar to \#2).

6. Similar to \#1, but with the lowest solar wind speed.

\subsection{Antisunward radial magnetic field and $V_{\alpha p}$}

To understand the variations in the $\alpha$ - protons relative speed $V_{\alpha p}$, we consider the transformation of the solar wind VDF at the magnetic field line kink created by a solar wind velocity shear. A simplified sketch of such a transformation is shown in Figure 6. This figure shows a hypothetical Z-like configuration appearing as a result of a velocity shear in the plasma. Such a configuration has been described by Gosling \& Skoug (2002), Owens et al. (2017) and Schwadron \& McComas (2021). The solar wind carrying the field line section " $F$ " is faster than the solar wind carrying the older field line section labeled "S." The 


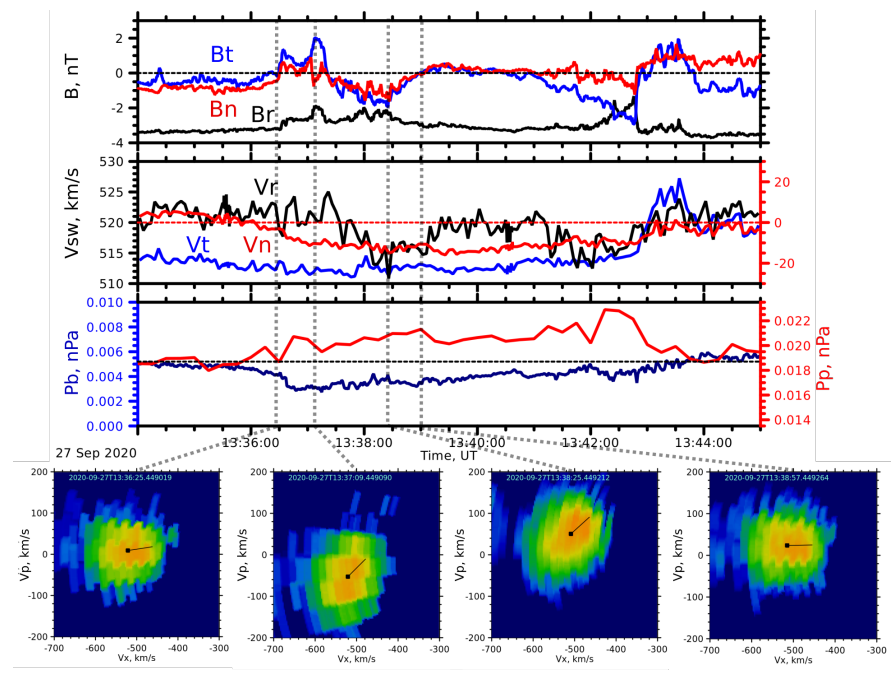

Fig. 5. Modification of the proton VDF at the small compressible pressure-balance perturbation. Details are similar to those in Figure 4. Second from top panel shows three velocity components and the third panel shows the magnetic field pressure (blue) and proton pressure (red) averaged for 15 seconds.

section labeled "I" connects two original field lines. The length of this section is increasing over time. Thus, the section "I" may only be filled with plasma via the two kinks connecting "F" with "I" (on the right in the diagram in Figure 6) and "S" with "I" (on the left). In the plasma frame, the left kink "I-S" is moving to the right (faster than the solar wind bulk) with the Alfven velocity. The original ion VDF, including $\alpha$-particles, in the section marked " $S$ " is shown by a green dashed contour on the left side of Figure 6. The center of the $\alpha$-particles distribution is shown as a small green circle. Originally, $V_{\alpha p}$, in the undisturbed fast solar wind, is positive ( $V_{\mathrm{He}^{++}}>V_{\mathrm{H}^{+}}$, see Marsch et al. (1982); Reisenfeld et al. (2001)). The part of original ion VDF on the left from the kink velocity (labeled "dHT") moves toward the kink and passes to the section "I." In this case, the particles' parallel velocity component changes sign in the kink velocity frame and becomes the part of the VDF shown in blue. We note that it is equivalent to the Walen test condition (Paschmann \& Daly 1998): there is no convection electric field in the kink frame of reference. Thus this "blue" distribution is a VDF that we would expedt to see in section "I." The bulk velocity of $\alpha$-particles is now less than the bulk velocity of the proton distribution. The same is true for the right kink connecting sections " $F$ " and "I" of the field line. It should be noted that: 1) in both cases, the $\alpha$ particles move slower than the protons on section "I"and 2) interesting, to allow for a sufficiently long life time of section "I," the kink "F"-'I" would move faster than kink "S"-'I," namely, $V_{F}-V_{S}>2 V_{A}$. Here, $V_{F}$ is the solar wind velocity at the "F" section, and $V_{S}$ is the solar wind velocity at the " $S$ " part. We assume that Alvfen velocity $V_{A}$ is more-or-less the same at both sides. It would be very natural to assume that the solar wind, observed at 09:30 UT (time interval \#1), when we see the positive (antisunward) $B_{r}$ and antiparallel strahl electrons, corresponds to section "I" of the magnetic field line. And it is likely that this field line is connected via a "F-I" kink to "F" section, carried with the maximal observed solar wind speed. We note that the negative $V_{\alpha p}$ seen at this time is consistent with the simple model in Figure 6.

The left panel of Figure 7 shows the VDF, observed at 09:30, with a small anisotropy $T_{\|} \lesssim T_{\perp}$. The same, albeit more pronounced anisotropy is seen in the proton VDF measured at 11:22:41 (see Figure 4, panel c) during a short magnetic field

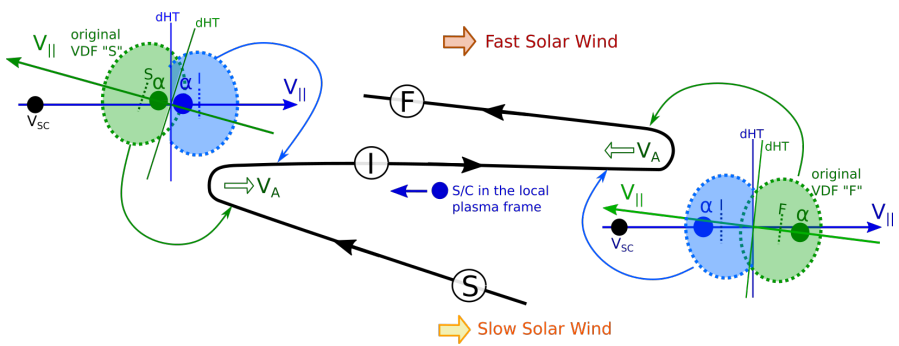

Fig. 6. Sketch explaining the transformation of the VDF and relative $V_{\alpha p}$ at the magnetic field line kinks of the switchbacks. The central diagram shows the magnetic line configuration containing three sections: "F" (top) in the fastest plasma stream, "S" (bottom) in the slowest plasma stream, and " $\mathrm{I}$ " the magnetic field section connecting the " $F$ " and "S" sections via two kinks. The blue dot under the "I" field line represents the spacecraft motion in the plasma frame of reference. The left and right diagrams show corresponding VDFs in the kink frame. The vertical solid line in the distribution shows the kink velocity (i.e. the deHoffman-Teller frame). The small dashed vertical lines in the distributions show the plasma bulk velocity in the kink frame. The green filled dashed contour shows the original proton distribution in sections "F" or "S", and the small dark green solid circle is the $\alpha$-particles bulk velocity. The blue filled contour and the dark blue circle are the part of the original VDF after the transformation at the kink.
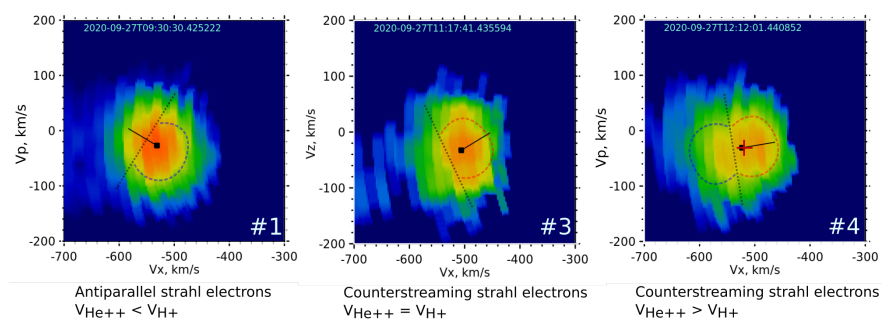

Fig. 7. Three examples of proton distribution functions in the plane containing $\boldsymbol{V}$ and $\boldsymbol{B}$ vectors. Left: Interval \#1, middle: Interval \#3, right: Interval \#4. The straight dashed lines show the position of the de Hoffmann-Teller frame origin, and the dashed contours show the parts of the proton VDFs passing the reconnection kink.

reversal to a antisunward direction. The distribution observed at 11:22:41 has the same bulk velocity ( taking into account the general velocity trend) and $T_{\|}<T_{\perp}$.

The middle panel of Figure 7 shows the VDF measured in region \#3 when the magnetic field was sunward. The solar wind velocity corresponds to the local minimum of the solar wind corridor in Figure 2, and $T_{\|}<T_{\perp}$. However, the $V_{\alpha p}$ is near zero and the strahl electrons are bidirectional. Such features do not correspond to expectations for antisunward magnetic filed line (type "S" in Figure 6). The right panel of Figure 7 shows the proton distribution for interval \#4 when the magnetic field is sunward, $V_{\alpha p}>0$, and solar wind velocity corresponds to the largest solar wind speed in Figure 2. It would correspond to a sunward field line (type "F") in our sketch, but the proton VDF is quite different from the VDF of an undisturbed solar wind. The present VDF contains two clearly distinguishable components. Our interpretation of this difference between the proton VDFs in regions \#3 and \#4 is that the corresponding magnetic field line reconnected upstream with the antisunward field line. The low energy part of ion VDF belonging to the antisunward field line (blue dashed contour in panel \#1) is mirroring (in the velocity space) relative to the de Hoffman-Teller frame origin while it passes from sunward line to antisunward one via the reconnection kink. We can see this in panel \#4 from the presence of a 

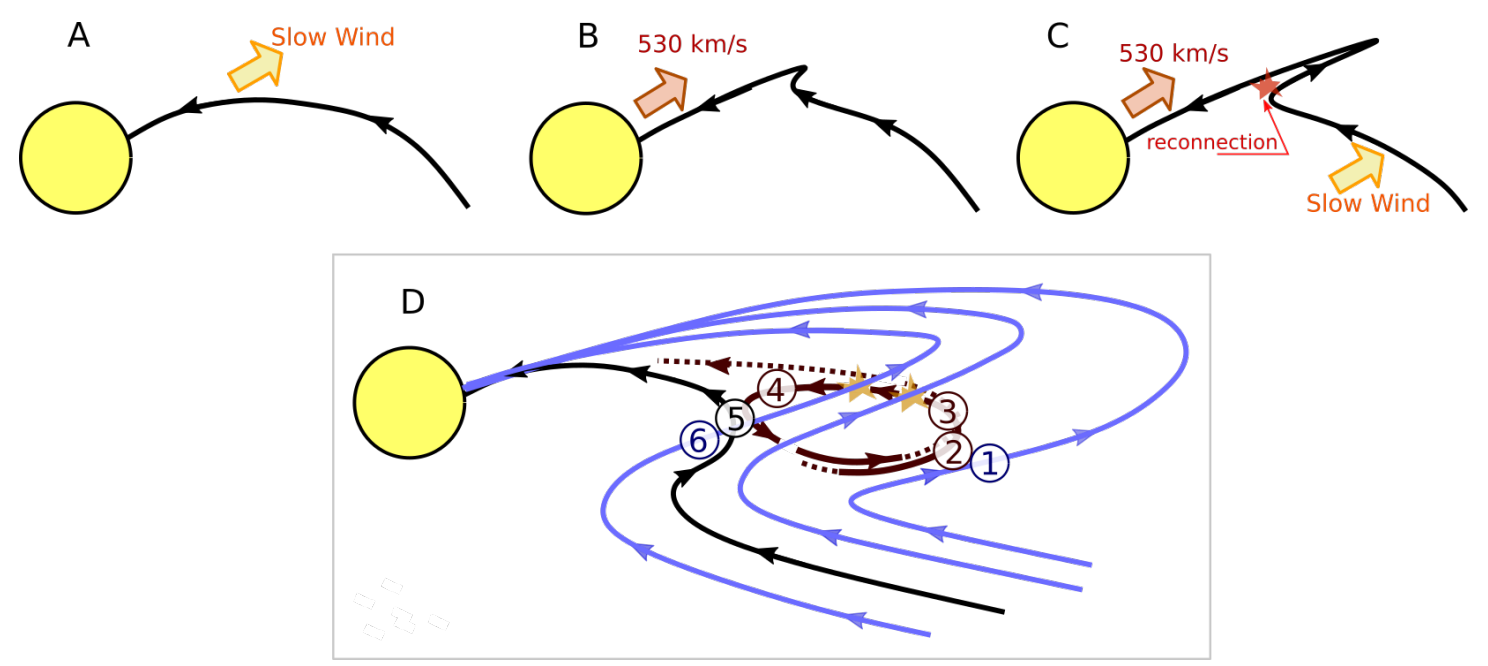

Fig. 8. Scenario of creation of the reversed open field line (A -C) and a flux rope (D) due to the solar wind velocity shear. The reversed but not reconnected field lines are shown in blue. The reconnected field line is shown in black. The detached part of the reconnected field line, creating a flux rope, is shown in brown. The numbers in the panel D correspond to the intervals in Figures 2 and 3 . See more details in the main text.

higher speed component in the compound VDF. Thus the accelerated part of the VDF from antisunward line is added to the original proton distribution on the corresponding sunward reconnected line (see Gosling et al. 2005). The mixture of two populations results in the bi-component distribution with increased bulk velocity.

\subsection{Event scenario}

The proposed scenario for the SolO observations in 9:00 - 16:30 time interval is similar to the magnetic field reversal ideas published by Gosling \& Skoug (2002); Owens et al. (2013, 2017), and later Schwadron \& McComas (2021). All these models require the footpoint of the field line to move across the photosphere and could cross the coronal hole boundary (see Fisk et al. 1999). If this happens, the velocity of the plasma belonging to this field line might change significantly. The popular model explaining such a motion is an interchange reconnection (see Fisk \& Kasper 2020; Owens et al. 2013; Kong et al. 2018). In this regard, Figure 7b of Owens et al. (2013) gives a very clear illustration of the formation of a "Z-like" line by an interchange reconnection. More recently, Schwadron \& McComas (2021) used the field line footpoint motion to explain both the radial interplanetary magnetic field and the "switchbacks". We try to use this approach in our scenario of the observed event.

Figure 8 shows the corresponding sketch. It assumes that the solar wind velocity at one given field line started to increase at a certain point in time. This leads to the progressive modification of the shape of the field line, as shown in the panels A-B-C. Such a configuration can be stable if the velocity shear is greater than the (roughly) sum of two local Alfven velocities at the location of the kinks. We then assume that the folded magnetic field lines of opposite directions can merge at some reconnection point as illustrated in panel "C." Such a reconnection leads to the formation of a rather large flux rope (Khabarova et al. 2015), as shown in brown in panel D. This model can explain two important observed features: 1) the bi-component $\left(T_{\|}>T_{\perp}\right.$ ) proton VDF in the sunward field line (see Section 3.2); and 2) the possible bi-directional strahl electrons. The last inference is slightly dubious, but we can assume that in the vicinity of the reconnection $\mathrm{X}$-line (Figure 8, D), the original antiparallel strahl electrons are moving toward the reconnection $\mathrm{X}$-line and some fraction of the electrons can pass from one thread to another via the electron scale's unmagnetized diffusion region (Burch et al. 2016; Chen et al. 2017) and then continuing to move parallel to the local magnetic field. Of course, we can see such electrons at the reconnection exhaust boundary only, but as we show below, SolO is always near the external boundary of the flux rope.

According to the sketch in panel D, the SolO observation sequence is summarized as follows (the items numbers correspond to the interval numbers in Figures 2, 3, and 8):

1. SolO samples an antisunward field line ( like section "I" in Figure 6), observing the negative $V_{\alpha p}$ and antiparallel strahl. The proton VDF has a small anisotropy with $T_{\|} \lesssim T_{\perp}$.

2. Time intervals \#2 and \#5 are very similar. SolO is in a high$\beta$ region where $B_{R}$ is very small, $B_{T}$ is positive, $V_{\alpha p} \approx 0$, and there are almost no strahl electrons. We can consider these zones as boundary layers likely resulting from additional reconnection between the blue antisunward field line and the sunward part of brown field line. Such a reconnection is shown by light yellow star in panel " $D$ ". The antisunward part of such reconnected field line is completely detached from Sun and lacks the strahl electrons.

3. SolO is in the sunward section of the magnetic flux rope, but not very close to the flux rope boundary. Here we observe a proton distribution with $T_{\|}<T_{\perp}$, and $V_{\alpha p} \gtrsim 0$. At the end of this time interval SolO exits the flux rope, and enters an antisunward field line (like section "I" in Figure 6) drawn in blue in Figure 8. Moreover, during this excursion the spacecraft is crossing two reconnection exhausts, created, possibly, by reconnections between the blue and brown field lines.

4. SolO is moving along the flux rope boundary observing short fluctuations of the magnetic field, which is possibly associated with the intermittent reconnection between brown and blue lines upstream. Here the proton VDF is very anisotropic with $T_{\|}>T_{\perp}$. The origin of such a distribution is shown in Figure 7 . We note that $B_{T}$ is negative at the and of the interval,

5. SolO is in a region of decreased magnetic field, increased density and higher proton velocity, consistent with a boundary layer formed by reconnection, similar to region \#2. We note that $B_{T}$ and $B_{R}$ are positive, that corresponds to the black field line in Figure 8. 
6. SolO is once again on the antisunward section of the magnetic field line (blue), and observes the same solar wind conditions as during interval \#1.

We note that in this scenario, SolO never touches the original low solar wind speed field line and we do not know the plasma bulk velocity in this case.

\subsection{Conclusions}

On 27 September 2020, Solar Orbiter sampled a solar wind stream magnetically connected to a southern hemisphere coronal hole. SolO was at $0.98 \mathrm{AU}$ from Sun. It observed a fast solar wind with strong fluctuations of the magnetic field and even clear reversals of $B_{r}$ with associated variations in the plasma flow. The suprathermal electrons pitch angle distribution and $\alpha$-particles' relative speed variations indicate that the magnetic field line was locally folded by $180^{\circ}$ as a result of velocity shear. Such a velocity shear at one magnetic field line can appear due to the line footprint motion from the streamer (or pseudostreamer) region to a coronal hole. This simple model can explain both the "switchbacks" observed by PSP on 27 September 2020 and the magnetic field inversions observed by SolO earlier that same day. However, the classic switchback " $Z$ " shape of the magnetic field line cannot explain all the features observed by SolO.

We observed proton VDFs with pronounced $T_{\|}>T_{\perp}$, whereas the original VDF is low anisotropic, with $T_{\|} \lesssim T_{\perp}$. Such a reconfiguration of the proton VDF is a consequence of the reconnection between two sections of the same folded magnetic field line. Such a scenario leads to formation of a flux rope, as shown in Figure 8. Thus, the structures observed by SolO may effectively stand as the surviving remains of the switchbacks created near Sun and also observed by PSP. However, unlike the typical Alfvenic fluctuation observed by PSP, after many reconnections, these structures have been converted to a large flux rope convecting with a velocity similar to the original velocity of the solar wind, created in the coronal hole and observed by PSP.

Acknowledgements. We would like to thank all the outstanding women and men who have made PAS, SWA and Solar Orbiter a reality, including the persons who contribute to the development of the software used for the analysis (CL, AMDA and CDPP). The French part of this work is supported by CNES and CNRS. Lubomir Prech and Tereza Durovcova were supported by Czech Science Fondation, grant no 19-18993S. Solar Orbiter magnetometer operations are funded by the UK Space Agency (grant ST/T001062/1). Tim Horbury is supported by STFC grant ST/S000364/1.

\section{References}

Altschuler, M. D. \& Newkirk, G. 1969, Solar Physics, 9, 131

Bale, S. D., Badman, S. T., Bonnell, J. W., et al. 2019, Nature, 576, 237

Bale, S. D., Goetz, K., Harvey, P. R., et al. 2016, Space Science Reviews, 204 49

Balogh, A., Forsyth, R. J., Lucek, E. A., Horbury, T. S., \& Smith, E. J. 1999, Geophysical Research Letters, 26, 631

Burch, J. L., Torbert, R. B., Phan, T. D., et al. 2016, Science, 352

Chen, L.-J., Hesse, M., Wang, S., et al. 2017, Journal of Geophysical Research: Space Physics, 122, 5235

Crooker, N. U., Burton, M. E., Siscoe, G. L., et al. 1996, J. Geophys. Res., 101, 24331

Crooker, N. U., Huang, C.-L., Lamassa, S. M., et al. 2004a, Journal of Geophysical Research: Space Physics, 109

Crooker, N. U., Kahler, S. W., Larson, D. E., \& Lin, R. P. 2004b, Journal of Geophysical Research: Space Physics, 109

Edmondson, J. K., Antiochos, S. K., DeVore, C. R., Lynch, B. J., \& Zurbuchen, T. H. 2010, The Astrophysical Journal, 714, 517

Elphic, R. C. 1990, Observations of Flux Transfer Events: Are Ftes Flux Ropes, Islands, or Surface Waves? (American Geophysical Union (AGU)), 455-471
Enžl, J., Přech, L., Šafránková, J., \& Němeček, Z. 2014, The Astrophysical Journal, 796, 21

Farrugia, C., Southwood, D., Cowley, S., Rijnbeek, R., \& Daly, P. 1987, Planetary and Space Science, 35, 737

Feldman, W. C., Asbridge, J. R., Bame, S. J., Montgomery, M. D., \& Gary, S. P. 1975, Journal of Geophysical Research (1896-1977), 80, 4181

Fisk, L. A. \& Kasper, J. C. 2020, The Astrophysical Journal, 894, L4

Fisk, L. A., Zurbuchen, T. H., \& Schwadron, N. A. 1999, The Astrophysical Journal, 521, 868

Fox, N. J., Velli, M. C., Bale, S. D., et al. 2016, Space Science Reviews, 204, 7

Froment, C., Krasnoselskikh, V., de Wit, T. D., et al. 2021, A\&A [arXiv:2101.06279]

García Marirrodriga, C., Pacros, A., Strandmoe, S., et al. 2021, A\&A, 646, A121

Gosling, J. T., Baker, D. N., Bame, S. J., et al. 1987, Journal of Geophysical Research: Space Physics, 92, 8519

Gosling, J. T. \& Skoug, R. M. 2002, Journal of Geophysical Research: Space Physics, 107, SSH 19

Gosling, J. T., Skoug, R. M., McComas, D. J., \& Smith, C. W. 2005, Journal of Geophysical Research: Space Physics, 110

Gosling, J. T., Tian, H., \& Phan, T. D. 2011, The Astrophysical Journal, 737, L35

Génot, V., Budnik, E., Jacquey, C., et al. 2021, Planetary and Space Science, 105214

Horbury, T. C., Laker, R., Woodham, L., et al. 2021, A\&A, this volume

Horbury, T. S., O’Brien, H., Carrasco Blazquez, I., et al. 2020, A\&A, 642, A9

Kahler, S. W., Crocker, N. U., \& Gosling, J. T. 1996, Journal of Geophysical Research: Space Physics, 101, 24373

Kasper, J. C., Abiad, R., Austin, G., et al. 2016, Space Science Reviews, 204, 131

Kasper, J. C., Bale, S. D., Belcher, J. W., et al. 2019, Nature, 576, 228

Khabarova, O., Zank, G. P., Li, G., et al. 2015, The Astrophysical Journal, 808 , 181

Kong, D. F., Pan, G. M., Yan, X. L., Wang, J. C., \& Li, Q. L. 2018, The Astrophysical Journal, 863, L22

Lavraud, B., Fargette, N., Reville, V., et al. 2020, The Astrophysical journal letters, 894, L19

Lepping, R. P., Jones, J. A., \& Burlaga, L. F. 1990, Journal of Geophysical Research: Space Physics, 95, 11957

Lockwood, M. 1995, Journal of Geophysical Research: Space Physics, 100, 21791

Lockwood, M., Owens, M., \& Rouillard, A. P. 2009, Journal of Geophysical Research: Space Physics, 114

Macneil, A. R., Owens, M. J., Lockwood, M., Štverák, Š., \& Owen, C. J. 2020, Solar Physics, 295, 16

Marsch, E., Muhlhauser, K.-H., Rosenbauer, H., Schwenn, R., \& Neubauer, F. M. 1982, Journal of Geophysical Research: Space Physics, 87, 35

Muller, D., St. Cyr, O. C., Zouganelis, I., et al. 2020, A\&A, 642, A1

Owen, C. J., Bruno, R., Livi, S., et al. 2020, A\&A, 642, A16

Owens, M. J., Crooker, N. U., \& Lockwood, M. 2013, Journal of Geophysical Research: Space Physics, 118, 1868

Owens, M. J., Lockwood, M., Riley, P., \& Linker, J. 2017, Journal of Geophysical Research: Space Physics, 122, 10,980

Paschmann, G. \& Daly, P. W. 1998, ISSI Scientific Reports Series, 1

Priest, E. R. 1990, The Equilibrium of Magnetic Flux Ropes (Tutorial Lecture) (American Geophysical Union (AGU)), 1-22

Reisenfeld, D. B., Gary, S. P., Gosling, J. T., et al. 2001, Journal of Geophysical Research: Space Physics, 106, 5693

Rouillard, A. P., Pinto, R. F., Vourlidas, A., et al. 2020, A\&A, 642, A2

Ruffolo, D., Matthaeus, W. H., Chhiber, R., et al. 2020, The Astrophysical Journal, 902, 94

Scholer, M. 1995, Models of Flux Transfer Events (American Geophysical Union (AGU)), 235-245

Schwadron, N. A. \& McComas, D. J. 2021, The Astrophysical Journal, 909, 95

Shodhan, S., Crooker, N. U., Kahler, S. W., et al. 2000, Journal of Geophysical Research: Space Physics, 105, 27261

Squire, J., Chandran, B. D. G., \& Meyrand, R. 2020, The Astrophysical Journal, 891, L2

Steinberg, J. T., Lazarus, A. J., Ogilvie, K. W., Lepping, R., \& Byrnes, J. 1996, Geophysical Research Letters, 23, 1183

Wiegelmann, T., Petrie, G. J. D., \& Riley, P. 2017, Space Science Reviews, 210, 249

Yamauchi, Y., Moore, R. L., Suess, S. T., Wang, H., \& Sakurai, T. 2004a, The Astrophysical Journal, 605, 511

Yamauchi, Y., Suess, S. T., \& Sakurai, T. 2002, Geophysical Research Letters, 29, 21

Yamauchi, Y., Suess, S. T., Steinberg, J. T., \& Sakurai, T. 2004b, Journal of Geophysical Research: Space Physics, 109 